\title{
EuGH: Urteil über Sampling in der Musikproduktion
}

\author{
EuGH Urteil vom 29.7.2019 - C-476/17
}

\section{ECLI:EU:C:2019:624}

- "Vorlage zur Vorabentscheidung - Urheberrecht und verwandte Schutzrechte - Richtlinie 2001/29/EG - Informationsgesellschaft - Harmonisierung bestimmter Aspekte des Urheberrechts und der verwandten Schutzrechte - Elektronisches Kopieren von Audiofragmenten (Sampling) - Art. 2 Buchst. c - Tonträgerhersteller - Vervielfältigungsrecht - ,Teilweise' Vervielfältigung - Art. 5 Abs. 2 und 3 - Ausnahmen und Beschränkungen - Reichweite - Art. 5 Abs. 3 Buchst. d - Zitate - Richtlinie 2006/115/EG - Art. 9 Abs. 1 Buchst. b - Verbreitungsrecht - Grundrechte - Charta der Grundrechte der Europäischen Union - Art. 13 - Freiheit der Kunst"

In der Rechtssache C476/17

betreffend ein Vorabentscheidungsersuchen nach Art. 267 AEUV, eingereicht vom Bundesgerichtshof (Deutschland) mit Entscheidung vom 1. Juni 2017, beim Gerichtshof eingegangen am 4. August 2017, in dem Verfahren

\section{Pelham GmbH,}

Moses Pelham,

\section{Martin Haas}

gegen

\section{Ralf Hütter,}

\section{Florian Schneider-Esleben}

erlässt der GERICHTSHOF (Große Kammer) unter Mitwirkung des Präsidenten K. Lenaerts, der Kammerpräsidenten A. Arabadjiev, M. Vilaras, und T. von Danwitz, der Kammerpräsidentin C. Toader, der Kammerpräsidenten F. Biltgen und C. Lycourgos sowie der Richter E. Juhász, M. Ilešič (Berichterstatter), L. Bay Larsen und S. Rodin, Generalanwalt: M. Szpunar, Kanzler: R. Șereș, Verwaltungsrätin, aufgrund des schriftlichen Verfahrens und auf die mündliche Verhandlung vom 3. Juli 2018, unter Berücksichtigung der Erklärungen

- der Pelham GmbH sowie von Herrn Pelham und Herrn Haas, vertreten durch Rechtsanwalt $A$. Walter,

- von Herrn Hütter und Herrn Schneider-Esleben, vertreten durch Rechtsanwältin U. Hundt-Neumann und Rechtsanwalt H. Lindhorst,

- der deutschen Regierung, vertreten durch T. Henze, M. Hellmann und J. Techert als Bevollmächtigte,
- der französischen Regierung, vertreten durch D. Colas, D. Segoin und E. Armoët als Bevollmächtigte,

- der Regierung des Vereinigten Königreichs, vertreten durch Z. Lavery und D. Robertson als Bevollmächtigte im Beistand von N. Saunders, Barrister,

- der Europäischen Kommission, vertreten durch T. Scharf und J. Samnadda als Bevollmächtigte,

nach Anhörung der Schlussanträge des Generalanwalts in der Sitzung vom 12. Dezember 2018 folgendes

\section{Urteil}

[1] Das Vorabentscheidungsersuchen betrifft die Auslegung von Art. 2 Buchst. c und Art. 5 Abs. 3 Buchst. d der Richtlinie 2001/29/EG des Europäischen Parlaments und des Rates vom 22. Mai 2001 zur Harmonisierung bestimmter Aspekte des Urheberrechts und der verwandten Schutzrechte in der Informationsgesellschaft (ABI. 2001, L 167, S. 10) sowie von Art. 9 Abs. 1 Buchst. b und Art. 10 Abs. 2 Unterabs. 1 der Richtlinie 2006/115/ EG des Europäischen Parlaments und des Rates vom 12. Dezember 2006 zum Vermietrecht und Verleihrecht sowie zu bestimmten dem Urheberrecht verwandten Schutzrechten im Bereich des geistigen Eigentums (ABI. 2006, L 376, S. 28).

[2] Es ergeht im Rahmen eines Rechtsstreits zwischen der Pelham GmbH, Herrn M. Pelham und Herrn M. Haas (im Folgenden zusammen: Pelham) auf der einen Seite sowie Herrn R. Hütter und Herrn F. Schneider-Esleben (im Folgenden: Hütter u.a.) auf der anderen Seite über die Nutzung einer etwa zweisekündigen Rhythmussequenz, die einem Tonträger der Musikgruppe Kraftwerk, deren Mitglieder Hütter u.a. sind, entnommen wurde, für die Aufnahme des Musiktitels "Nur mir", der von Herrn Pelham und Herrn Haas komponiert und von der Pelham GmbH produziert wurde.

\section{Rechtlicher Rahmen}

\section{Internationales Recht}

[3] Art. 1 des am 29. Oktober 1971 in Genf unterzeichneten Übereinkommens zum Schutz der Hersteller von Tonträgern gegen die unerlaubte Vervielfältigung ihrer Tonträger (im Folgenden: Genfer Übereinkommen) lautet:

„Für die Zwecke dieses Übereinkommens versteht man unter

a) ,Tonträger' jede ausschließlich auf den Ton beschränkte Festlegung der Töne einer Darbietung oder anderer Töne; 
b) ,Hersteller von Tonträgern' die natürliche oder juristische Person, die zum ersten Mal die Töne einer Darbietung oder andere Töne festlegt;

c) ,Vervielfältigungsstück' einen Gegenstand, der einem Tonträger unmittelbar oder mittelbar entnommene Töne enthält und der alle oder einen wesentlichen Teil der in dem Tonträger festgelegten Töne verkörpert;

d) ,Verbreitung an die Öffentlichkeit' jede Handlung, durch die Vervielfältigungsstücke eines Tonträgers der Allgemeinheit oder einem Teil der Allgemeinheit unmittelbar oder mittelbar angeboten werden."

[4] Art. 2 dieses Übereinkommens bestimmt:

„Jeder Vertragsstaat schützt die Hersteller von Tonträgern, die Angehörige anderer Vertragsstaaten sind, gegen die Herstellung von Vervielfältigungsstücken ohne Zustimmung des Herstellers des Tonträgers und gegen die Einfuhr solcher Vervielfältigungsstücke, sofern die Herstellung oder die Einfuhr zum Zweck der Verbreitung an die Öffentlichkeit erfolgt, und auch gegen die Verbreitung solcher Vervielfältigungsstücke an die Öffentlichkeit."

\section{Unionsrecht}

\section{Richtlinie 2001/29}

[5] In den Erwägungsgründen 3, 4, 6, 7, 9, 10, 31 und 32 der Richtlinie 2001/29 heißt es:

„(3) Die vorgeschlagene Harmonisierung trägt zur Verwirklichung der vier Freiheiten des Binnenmarkts bei und steht im Zusammenhang mit der Beachtung der tragenden Grundsätze des Rechts, insbesondere des Eigentums einschließlich des geistigen Eigentums, der freien Meinungsäußerung und des Gemeinwohls.

(4) Ein harmonisierter Rechtsrahmen zum Schutz des Urheberrechts und der verwandten Schutzrechte wird durch erhöhte Rechtssicherheit und durch die Wahrung eines hohen Schutzniveaus im Bereich des geistigen Eigentums substanzielle Investitionen in Kreativität und Innovation einschließlich der Netzinfrastruktur fördern und somit zu Wachstum und erhöhter Wettbewerbsfähigkeit der europäischen Industrie beitragen, und zwar sowohl bei den Inhalten und der Informationstechnologie als auch allgemeiner in weiten Teilen der Industrie und des Kultursektors. Auf diese Weise können Arbeitsplätze erhalten und neue Arbeitsplätze geschaffen werden. [...]

(6) Ohne Harmonisierung auf Gemeinschaftsebene könnten Gesetzgebungsinitiativen auf einzelstaatlicher Ebene, die in einigen Mitgliedstaaten bereits in die Wege geleitet worden sind, um den technischen Herausforderungen zu begeg- nen, erhebliche Unterschiede im Rechtsschutz und dadurch Beschränkungen des freien Verkehrs von Dienstleistungen und Produkten mit urheberrechtlichem Gehalt zur Folge haben, was zu einer Zersplitterung des Binnenmarkts und zu rechtlicher Inkohärenz führen würde. Derartige rechtliche Unterschiede und Unsicherheiten werden sich im Zuge der weiteren Entwicklung der Informationsgesellschaft, in deren Gefolge die grenzüberschreitende Verwertung des geistigen Eigentums bereits stark zugenommen hat, noch stärker auswirken. Diese Entwicklung wird und sollte fortschreiten. Erhebliche rechtliche Unterschiede und Unsicherheiten in Bezug auf den Rechtsschutz können die Erzielung von GröBenvorteilen für neue Produkte und Dienstleistungen mit urheber- und leistungsschutzrechtlichem Gehalt beschränken.

(7) Der bestehende Gemeinschaftsrechtsrahmen zum Schutz des Urheberrechts und der verwandten Schutzrechte ist daher anzupassen und zu ergänzen, soweit dies für das reibungslose Funktionieren des Binnenmarkts erforderlich ist. Zu diesem Zweck sollten diejenigen einzelstaatlichen Rechtsvorschriften über das Urheberrecht und die verwandten Schutzrechte, die sich von Mitgliedstaat zu Mitgliedstaat beträchtlich unterscheiden oder eine derartige Rechtsunsicherheit bewirken, dass der Binnenmarkt in seiner Funktionsfähigkeit beeinträchtigt und die Informationsgesellschaft in Europa in ihrer Entwicklung behindert wird, angepasst und uneinheitliches Vorgehen der Mitgliedstaaten gegenüber technischen Entwicklungen vermieden werden, während Unterschiede, die das Funktionieren des Binnenmarkts nicht beeinträchtigen, nicht beseitigt oder verhindert zu werden brauchen. [...]

(9) Jede Harmonisierung des Urheberrechts und der verwandten Schutzrechte muss von einem hohen Schutzniveau ausgehen, da diese Rechte für das geistige Schaffen wesentlich sind. Ihr Schutz trägt dazu bei, die Erhaltung und Entwicklung kreativer Tätigkeit im Interesse der Urheber, ausübenden Künstler, Hersteller, Verbraucher, von Kultur und Wirtschaft sowie der breiten Öffentlichkeit sicherzustellen....

(10) Wenn Urheber und ausübende Künstler weiter schöpferisch und künstlerisch tätig sein sollen, müssen sie für die Nutzung ihrer Werke eine angemessene Vergütung erhalten, was ebenso für die Produzenten gilt, damit diese die Werke finanzieren können. Um Produkte wie Tonträger, Filme oder Multimediaprodukte herstellen und Dienstleistungen, z. B. Dienste auf Abruf, anbieten zu können, sind beträchtliche Investitionen erforderlich. Nur wenn die Rechte des geistigen Eigentums angemessen geschützt werden, kann eine angemessene Vergütung der Rechtsinhaber gewährleistet und ein zufrieden stellender Ertrag dieser Investitionen sichergestellt werden. [...] 
(31) Es muss ein angemessener Rechts- und Interessenausgleich zwischen den verschiedenen Kategorien von Rechtsinhabern sowie zwischen den verschiedenen Kategorien von Rechtsinhabern und Nutzern von Schutzgegenständen gesichert werden. Die von den Mitgliedstaaten festgelegten Ausnahmen und Beschränkungen in Bezug auf Schutzrechte müssen vor dem Hintergrund der neuen elektronischen Medien neu bewertet werden. ... Um ein reibungsloses Funktionieren des Binnenmarkts zu gewährleisten, sollten diese Ausnahmen und Beschränkungen einheitlicher definiert werden. Dabei sollte sich der Grad ihrer Harmonisierung nach ihrer Wirkung auf die Funktionsfähigkeit des Binnenmarkts bestimmen.

(32) Die Ausnahmen und Beschränkungen in Bezug auf das Vervielfältigungsrecht und das Recht der öffentlichen Wiedergabe sind in dieser Richtlinie erschöpfend aufgeführt. Einige Ausnahmen oder Beschränkungen gelten, soweit dies angemessen erscheint, nur für das Vervielfältigungsrecht. Diese Liste trägt den unterschiedlichen Rechtstraditionen in den Mitgliedstaaten Rechnung und soll gleichzeitig die Funktionsfähigkeit des Binnenmarkts sichern. Die Mitgliedstaaten sollten diese Ausnahmen und Beschränkungen in kohärenter Weise anwenden; dies wird bei der zukünftigen Überprüfung der Umsetzungsvorschriften besonders berücksichtigt werden."

[6] Art. 2 („Vervielfältigungsrecht“) der Richtlinie 2001/29 bestimmt:

„Die Mitgliedstaaten sehen für folgende Personen das ausschließliche Recht vor, die unmittelbare oder mittelbare, vorübergehende oder dauerhafte Vervielfältigung aufjede Art und Weise und in jeder Form ganz oder teilweise zu erlauben oder zu verbieten: [...]

c) für die Tonträgerhersteller in Bezug auf ihre Tonträger, ..."

[7] Art. 5 dieser Richtlinie sieht Ausnahmen und Beschränkungen für die in den Art. 2 bis 4 der Richtlinie genannten Rechte vor. In den Abs. 3 und 5 heißt es:

„(3) Die Mitgliedstaaten können in den folgenden Fällen Ausnahmen oder Beschränkungen in Bezug auf die in den Art. 2 und 3 vorgesehenen Rechte vorsehen: [...]

d) für Zitate zu Zwecken wie Kritik oder Rezensionen, sofern sie ein Werk oder einen sonstigen Schutzgegenstand betreffen, das bzw. der der Öffentlichkeit bereits rechtmäßig zugänglich gemacht wurde, sofern - außer in Fällen, in denen sich dies als unmöglich erweist - die Quelle, einschließlich des Namens des Urhebers, angegeben wird und sofern die Nutzung den anständigen Gepflogenheiten entspricht und in ihrem Umfang durch den besonderen Zweck gerechtfertigt ist; [...]
(5) Die in den Abs. 1, 2, 3 und 4 genannten Ausnahmen und Beschränkungen dürfen nur in bestimmten Sonderfällen angewandt werden, in denen die normale Verwertung des Werks oder des sonstigen Schutzgegenstands nicht beeinträchtigt wird und die berechtigten Interessen des Rechtsinhabers nicht ungebührlich verletzt werden."

\section{Richtlinie 2006/115}

[8] In den Erwägungsgründen 2, 5 und 7 der Richtlinie 2006/115 heißt es:

„(2) Das Vermieten und Verleihen von urheberrechtlich geschützten Werken und Gegenständen der verwandten Schutzrechte spielt insbesondere für die Urheber und die ausübenden Künstler sowie für die Hersteller von Tonträgern und Filmen eine immer wichtigere Rolle. Die Piraterie stellt eine zunehmende Bedrohung dar. [...]

(5) Um ihre Tätigkeit ausüben zu können, bedürfen Urheber und ausübende Künstler eines angemessenen Einkommens als Grundlage für weiteres schöpferisches und künstlerisches Arbeiten. Die insbesondere für die Herstellung von Tonträgern und Filmen erforderlichen Investitionen sind außerordentlich hoch und risikoreich. Die Möglichkeit, ein solches Einkommen sicherzustellen und solche Investitionen abzusichern, kann nur durch einen angemessenen Rechtsschutz für die jeweils betroffenen Rechtsinhaber wirkungsvoll gewährleistet werden. [...]

(7) Die Angleichung der Rechtsvorschriften der Mitgliedstaaten sollte in der Weise erfolgen, dass die Rechtsvorschriften nicht in Widerspruch zu den internationalen Übereinkommen stehen, auf denen das Urheberrecht und die verwandten Schutzrechte in vielen Mitgliedstaaten beruhen."

[9] Art. 1 („Regelungszweck“) dieser Richtlinie bestimmt in Abs. 1:

„In Übereinstimmung mit den Bestimmungen dieses Kapitels sehen die Mitgliedstaaten vorbehaltlich Art. 6 das Recht vor, die Vermietung und das Verleihen von Originalen und Vervielfältigungsstücken urheberrechtlich geschützter Werke und anderer in Art. 3 Abs. 1 bezeichneter Schutzgegenstände zu erlauben oder zu verbieten."

[10] Art. 9 („Verbreitungsrecht $\left.{ }^{\prime \prime}\right)$ der Richtlinie sieht in Abs. 1 vor:

„Die Mitgliedstaaten sehen das ausschließliche Recht, die in den Buchstaben a bis d genannten Schutzgegenstände sowie Kopien davon der Öffentlichkeit im Wege der Veräußerung oder auf sonstige Weise zur Verfügung zu stellen (nachstehend,Verbreitungsrecht' genannt), wie folgt vor: [...]

b) für Tonträgerhersteller in Bezug auf ihre Tonträger; ..." 
[11] In Art. 10 Abs. 2 Unterabs. 1 der Richtlinie 2006/115 heißt es: „... jeder Mitgliedstaat [kann] für den Schutz der ausübenden Künstler, Tonträgerhersteller, Sendeunternehmen und Hersteller der erstmaligen Aufzeichnungen von Filmen Beschränkungen der gleichen Art vorsehen, wie sie für den Schutz des Urheberrechts an Werken der Literatur und der Kunst vorgesehen sind."

\section{Deutsches Recht}

[12] Das Gesetz über Urheberrecht und verwandte Schutzrechte - Urheberrechtsgesetz vom 9. September 1965 (BGBI. I S. 1273, im Folgenden: UrhG) bestimmt in § 24:

„(1) Ein selbständiges Werk, das in freier Benutzung des Werkes eines anderen geschaffen worden ist, darf ohne Zustimmung des Urhebers des benutzten Werkes veröffentlicht und verwertet werden.

(2) Abs. 1 gilt nicht für die Benutzung eines Werkes der Musik, durch welche eine Melodie erkennbar dem Werk entnommen und einem neuen Werk zugrunde gelegt wird."

[13] 85 Abs. 1 UrhG, mit dem Art. 2 Buchst. c der Richtlinie 2001/29 und Art. 9 der Richtlinie 2006/115 umgesetzt werden, sieht in Satz 1 Fall 1 und 2 vor, dass der Hersteller eines Tonträgers das ausschließliche Recht hat, den Tonträger zu vervielfältigen und zu verbreiten.

\section{Ausgangsverfahren und Vorlagefragen}

[14] Hütter u.a. sind Mitglieder der Musikgruppe Kraftwerk. Diese veröffentlichte im Jahr 1977 einen Tonträger, auf dem sich das Musikstück "Metall auf Metall“ befindet.

[15] Herr Pelham und Herr Haas sind die Komponisten des Musikstücks „Nur mir“, das im Jahr 1997 auf Tonträgern der Pelham $\mathrm{GmbH}$ erschienen ist.

[16] Hütter u.a. tragen vor, Pelham habe etwa zwei Sekunden einer Rhythmussequenz aus dem Titel "Metall auf Metall“ elektronisch kopiert ("gesampelt") und dem Titel "Nur mir" in fortlaufender Wiederholung unterlegt, obwohl es ihnen möglich gewesen wäre, die übernommene Rhythmussequenz selbst einzuspielen.

[17] Sie sind der Auffassung, dass Pelham ihr Leistungsschutzrecht als Tonträgerhersteller verletzt habe. Hilfsweise machen sie geltend, das ihnen als ausübende Künstler zustehende Recht am geistigen Eigentum sowie das Urheberrecht von Herrn Hütter an dem musikalischen Werk seien verletzt worden. Äußerst hilfsweise tragen sie vor, dass Pelham gegen das Wettbewerbsrecht verstoßen habe.
[18] Hütter u.a. erhoben Klage beim Landgericht Hamburg (Deutschland) und beantragten Unterlassung, Schadensersatz, Auskunftserteilung und Herausgabe der Tonträger zum Zweck ihrer Vernichtung.

[19] Das Landgericht Hamburg gab der Klage statt. Die Berufung von Pelham beim OLG Hamburg (Deutschland) wurde zurückgewiesen. Auf die Revision von Pelham zum BGH (Deutschland) wurde das Urteil des OLG Hamburg aufgehoben und die Sache zu erneuter Prüfung an dieses Gericht zurückverwiesen. Das OLG Hamburg wies die Berufung von Pelham abermals zurück. Mit Urteil vom 13. Dezember 2012 wies der BGH die erneute Revision von Pelham zurück. Dieses Urteil wurde vom BVerfG (Deutschland) aufgehoben, das die Sache an das vorlegende Gericht zurückverwies.

[20] Das vorlegende Gericht führt aus, der Erfolg der Revision hänge von der Auslegung von Art. 2 Buchst. c und Art. 5 Abs. 3 Buchst. d der Richtlinie 2001/29 sowie Art. 9 Abs. 1 Buchst. b und Art. 10 Abs. 2 der Richtlinie 2006/115 ab.

[21] Erstens sei zu ermitteln, ob Pelham durch die Nutzung der Tonaufnahme von Hütter u.a. bei der Realisierung des eigenen Tonträgers in deren ausschließliches Recht eingegriffen habe, den Tonträger, auf dem sich der Titel "Metall auf Metall" befinde, iSv $\S 85$ Abs. 1 UrhG, mit dem Art. 2 Buchst. c der Richtlinie 2001/29 und Art. 9 der Richtlinie 2006/115 umgesetzt würden, zu vervielfältigen und zu verbreiten. Insbesondere sei zu klären, ob ein solcher Eingriff festgestellt werden könne, wenn einem Tonträger, wie im vorliegenden Fall, zwei Sekunden einer Rhythmussequenz entnommen und auf einen anderen Tonträger übertragen würden, und ob es sich bei diesem Tonträger um eine Kopie eines anderen Tonträgers iSv Art. 9 Abs. 1 Buchst. b der Richtlinie 2006/115 handele.

[22] Zweitens stelle sich für den Fall, dass ein solcher Eingriff in das Recht des Tonträgerherstellers festgestellt werde, die Frage, ob sich Pelham mit Erfolg auf das Recht zur freien Benutzung nach § 24 Abs. 1 UrhG berufen könne, das entsprechend für das Recht des Tonträgerherstellers gelte, wonach ein selbständiges Werk, das in freier Benutzung des Werkes eines anderen geschaffen worden sei, ohne Zustimmung des Urhebers des benutzten Werkes veröffentlicht und verwertet werden dürfe. Für diese Bestimmung gebe es keine ausdrückliche Entsprechung im Unionsrecht. Es stelle sich daher die Frage, ob sie mit dem Unionsrecht vereinbar sei, denn sie beschränke den Schutzbereich des ausschließlichen Rechts des Tonträgerherstellers zur Vervielfältigung und Verbreitung seines Tonträgers.

[23] Drittens beruhten die im nationalen Recht vorgesehenen Ausnahmen und Beschränkungen in Bezug auf das Vervielfältigungsrecht aus Art. 2 Buchst. c der Richtlinie 2001/29 und das Verbreitungsrecht aus Art. 9 Abs. 1 Buchst. b der Richtlinie 2006/115 auf Art. 5 Abs. 3 der Richtlinie 2001/29 und auf Art. 10 
Abs. 2 Unterabs. 1 der Richtlinie 2006/115. Es bestünden Zweifel hinsichtlich der Auslegung dieser Bestimmungen unter Umständen wie denen des Ausgangsverfahrens.

[24] Viertens seien die einschlägigen Bestimmungen des Unionsrechts unter Berücksichtigung der durch die Charta der Grundrechte der Europäischen Union (im Folgenden: Charta) garantierten Grundrechte auszulegen und anzuwenden. Dabei stelle sich die Frage, ob die Mitgliedstaaten bei der Umsetzung von Art. 2 Buchst. c und Art. 5 Abs. 2 und 3 der Richtlinie 2001/29 sowie von Art. 9 Abs. 1 Buchst. b und Art. 10 Abs. 2 Unterabs. 1 der Richtlinie 2006/115 in nationales Recht über einen Gestaltungsspielraum verfügten. Nach der Rechtsprechung des Bundesverfassungsgerichts seien nämlich innerstaatliche Rechtsvorschriften, die eine Richtlinie der Europäischen Union umsetzten, grundsätzlich nicht am Maßstab der Grundrechte des Grundgesetzes für die Bundesrepublik Deutschland vom 23. Mai 1949 (BGBI. I S. 1), sondern allein an den durch das Unionsrecht gewährleisteten Grundrechten zu messen, soweit die Richtlinie den Mitgliedstaaten keinen Umsetzungsspielraum überlasse. Im Übrigen bestünden unter Umständen wie denen des Ausgangsverfahrens Zweifel hinsichtlich der Auslegung dieser Grundrechte.

[25] Unter diesen Umständen hat der Bundesgerichtshof beschlossen, das Verfahren auszusetzen und dem Gerichtshof folgende Fragen zur Vorabentscheidung vorzulegen:

1. Liegt ein Eingriff in das ausschließliche Recht des Tonträgerherstellers zur Vervielfältigung seines Tonträgers aus Art. 2 Buchst. c der Richtlinie 2001/29 vor, wenn seinem Tonträger kleinste Tonfetzen entnommen und auf einen anderen Tonträger übertragen werden?

2. Handelt es sich bei einem Tonträger, der von einem anderen Tonträger übertragene kleinste Tonfetzen enthält, im Sinne von Art. 9 Abs. 1 Buchst. b der Richtlinie 2006/115 um eine Kopie des anderen Tonträgers?

3. Können die Mitgliedstaaten eine Bestimmung vorsehen, die - wie die Bestimmung des $\S 24$ Abs. 1 UrhG - klarstellt, dass der Schutzbereich des ausschließlichen Rechts des Tonträgerherstellers zur Vervielfältigung (Art. 2 Buchst. c der Richtlinie 2001/29) und Verbreitung (Art. 9 Abs. 1 Buchst. b der Richtlinie 2006/115) seines Tonträgers in der Weise immanent beschränkt ist, dass ein selbständiges Werk, das in freier Benutzung seines Tonträgers geschaffen worden ist, ohne seine Zustimmung verwertet werden darf?

4. Wird ein Werk oder ein sonstiger Schutzgegenstand iSv Art. 5 Abs. 3 Buchst. d der Richtlinie 2001/29 für Zitatzwecke genutzt, wenn nicht erkennbar ist, dass ein fremdes Werk oder ein fremder sonstiger Schutzgegenstand genutzt wird?
5. Lassen die Vorschriften des Unionsrechts zum Vervielfältigungsrecht und Verbreitungsrecht des Tonträgerherstellers (Art. 2 Buchst. c der Richtlinie 2001/29 und Art. 9 Abs. 1 Buchst. b der Richtlinie 2006/115) und den Ausnahmen oder Beschränkungen dieser Rechte (Art. 5 Abs. 2 und 3 der Richtlinie 2001/29 und Art. 10 Abs. 2 Unterabs. 1 der Richtlinie 2006/115) Umsetzungsspielräume im nationalen Recht?

6. In welcher Weise sind bei der Bestimmung des Schutzumfangs des ausschließlichen Rechts des Tonträgerherstellers zur Vervielfältigung (Art. 2 Buchst. c der Richtlinie 2001/29) und Verbreitung (Art. 9 Abs. 1 Buchst. b der Richtlinie 2006/115) seines Tonträgers und der Reichweite der Ausnahmen oder Beschränkungen dieser Rechte (Art. 5 Abs. 2 und 3 der Richtlinie 2001/29 und Art. 10 Abs. 2 Unterabs. 1 der Richtlinie 2006/115) die Grundrechte der Charta zu berücksichtigen?

\section{Zu den Vorlagefragen}

\section{Zur ersten und zur sechsten Frage}

[26] Mit seiner ersten und seiner sechsten Frage, die zusammen zu prüfen sind, möchte das vorlegende Gericht wissen, ob Art. 2 Buchst. c der Richtlinie 2001/29 unter Berücksichtigung der Charta dahin auszulegen ist, dass das ausschließliche Recht des Tonträgerherstellers, die Vervielfältigung seines Tonträgers zu erlauben oder zu verbieten, ihm gestattet, sich dagegen zu wehren, dass ein Dritter seinem Tonträger ein - auch nur sehr kurzes - Audiofragment entnimmt, um es in einen anderen Tonträger einzufügen.

[27] Nach Art. 2 Buchst. c der Richtlinie 2001/29 sehen die Mitgliedstaaten für die Tonträgerhersteller in Bezug auf ihre Tonträger das ausschließliche Recht vor, "die unmittelbare oder mittelbare, vorübergehende oder dauerhafte Vervielfältigung auf jede Art und Weise und in jeder Form ganz oder teilweise zu erlauben oder zu verbieten".

[28] Die Richtlinie 2001/29 definiert nicht, was unter der Wendung „Vervielfältigung ... ganz oder teilweise“ im Sinne dieser Bestimmung zu verstehen ist. Ihre Bedeutung und Tragweite ist daher nach ständiger Rechtsprechung des Gerichtshofs entsprechend ihrem Sinn nach dem gewöhnlichen Sprachgebrauch zu bestimmen, wobei zu berücksichtigen ist, in welchem Zusammenhang sie verwendet wird und welche Ziele mit der Regelung verfolgt werden, zu der sie gehört (Urteil vom 3. September 2014, Deckmyn und Vrijheidsfonds, C201/13, EU:C:2014:2132, Rn. 19 und die dort angeführte Rechtsprechung).

[29] Aus dem in Rn. 27 des vorliegenden Urteils wiedergegebenen Wortlaut von Art. 2 Buchst. c der Richtlinie 2001/29 ergibt sich, dass die Vervielfältigung eines - auch nur sehr kurzen Audiofragments eines Tonträgers durch einen Nutzer grundsätzlich als eine "teilweise" Vervielfältigung dieses Tonträgers 
im Sinne dieser Bestimmung anzusehen ist und eine solche Vervielfältigung somit unter das ausschließliche Recht des Tonträgerherstellers aus dieser Bestimmung fällt.

[30] Diese wörtliche Auslegung von Art. 2 Buchst. c der Richtlinie 2001/29 entspricht sowohl dem allgemeinen Ziel dieser Richtlinie, das, wie sich aus ihren Erwägungsgründen 4, 9 und 10 ergibt, darin besteht, ein hohes Schutzniveau für das Urheberrecht und die verwandten Schutzrechte zu erreichen, als auch dem im zehnten Erwägungsgrund genannten spezifischen Ziel des ausschließlichen Rechts des Tonträgerherstellers, seine Investitionen zu schützen. Wie nämlich ebenfalls im zehnten Erwägungsgrund ausgeführt, sind beträchtliche Investitionen erforderlich, um Produkte wie Tonträger anbieten zu können, so dass den Tonträgerherstellern die Möglichkeit zu geben ist, einen zufriedenstellenden Ertrag zu erzielen.

[31] Entnimmt jedoch ein Nutzer in Ausübung der Kunstfreiheit einem Tonträger ein Audiofragment, um es in geänderter und beim Hören nicht wiedererkennbarer Form in einem neuen Werk zu nutzen, stellt eine solche Nutzung keine „Vervielfältigung" iSv Art. 2 Buchst. c der Richtlinie 2001/29 dar.

[32] Insoweit ist darauf hinzuweisen, dass nach den Erwägungsgründen 3 und 31 der Richtlinie 2001/29 die durch diese Richtlinie bewirkte Harmonisierung insbesondere vor dem Hintergrund der elektronischen Medien einen angemessenen Ausgleich zwischen den Interessen der Inhaber von Urheber- und verwandten Schutzrechten am Schutz ihres nun in Art. 17 Abs. 2 der Charta verankerten Rechts am geistigen Eigentum auf der einen Seite und dem Schutz der Interessen und Grundrechte der Nutzer von Schutzgegenständen sowie dem Allgemeininteresse auf der anderen Seite sichern soll (vgl. in diesem Sinne Urteil vom 7. August 2018, Renckhoff, C161/17, EU:C:2018:634, Rn. 41).

[33] So hat der Gerichtshof bereits entschieden, dass sich weder aus Art. 17 Abs. 2 der Charta noch aus der Rechtsprechung des Gerichtshofs ergibt, dass das in dieser Bestimmung verankerte Recht des geistigen Eigentums schrankenlos und sein Schutz daher bedingungslos zu gewährleisten wäre (Urteile vom 24. November 2011, Scarlet Extended, C70/10, EU:C:2011:771, Rn. 43, vom 16. Februar 2012, SABAM, C360/10, EU:C:2012:85, Rn. 41, und vom 27. März 2014, UPC Telekabel Wien, C314/12, EU:C:2014:192, Rn. 61).

[34] Dieses Recht muss nämlich gegen die anderen Grundrechte abgewogen werden, darunter die durch Art. 13 der Charta garantierte Freiheit der Kunst, die es ermöglicht, am öffentlichen Austausch von kulturellen, politischen und sozialen Informationen und Ideen aller Art teilzuhaben, weil sie zur Freiheit der Meinungsäußerung gehört, die durch Art. 11 der Charta und Art. 10 Abs. 1 der am 4. November 1950 in Rom unterzeichneten Europäischen Konvention zum Schutz der Menschenrechte und Grundfreiheiten geschützt ist (vgl. in diesem Sinne EGMR, 24. Mai 1988, Müller u.a./Schweiz, CE:ECHR:1988:0524JUD001073784, § 27, EGMR, 8. Juli 1999, Karataş/Türkei, CE:ECHR:1999:0708JUD002316894, § 49).

[35] Insoweit ist festzustellen, dass die Technik des „Elektronischen Kopierens von Audiofragmenten“ (Sampling), bei der ein Nutzer - zumeist mit Hilfe elektronischer Geräte - einem Tonträger ein Audiofragment entnimmt und dieses zur Schaffung eines neuen Werks nutzt, eine künstlerische Ausdrucksform ist, die unter die durch Art. 13 der Charta geschützte Freiheit der Kunst fällt.

[36] In Ausübung dieser Freiheit kann der Nutzer eines Audiofragments (Sample) bei der Schaffung eines neuen Werks das dem Tonträger entnommene Fragment so ändern, dass es im neuen Werk beim Hören nicht wiedererkennbar ist.

[37] Die Annahme, dass ein Audiofragment, das für das eigene künstlerische Schaffen einem Tonträger entnommen und in einem neuen Werk in geänderter und beim Hören nicht wiedererkennbarer Form genutzt wird, eine "Vervielfältigung" dieses Tonträgers iSv Art. 2 Buchst. c der Richtlinie 2001/29 darstellt, widerspräche nicht nur dem Sinn dieses Begriffs nach dem gewöhnlichen Sprachgebrauch im Sinne der in Rn. 28 des vorliegenden Urteils angeführten Rechtsprechung, sondern missachtete auch das in Rn. 32 des vorliegenden Urteils angeführte Erfordernis eines angemessenen Ausgleichs.

[38] Eine solche Auslegung würde es dem Tonträgerhersteller insbesondere ermöglichen, sich in dem geschilderten Fall dagegen zu wehren, dass ein Dritter zum Zweck des künstlerischen Schaffens ein - auch nur sehr kurzes - Audiofragment aus seinem Tonträger entnimmt, obwohl eine solche Entnahme ihm nicht die Möglichkeit nimmt, einen zufriedenstellenden Ertrag aus seinen Investitionen zu erzielen.

[39] Nach alledem ist auf die erste und die sechste Frage zu antworten, dass Art. 2 Buchst. c der Richtlinie 2001/29 unter Berücksichtigung der Charta dahin auszulegen ist, dass das ausschließliche Recht des Tonträgerherstellers aus dieser Bestimmung, die Vervielfältigung seines Tonträgers zu erlauben oder zu verbieten, ihm gestattet, sich dagegen zu wehren, dass ein Dritter ein - auch nur sehr kurzes - Audiofragment seines Tonträgers nutzt, um es in einen anderen Tonträger einzufügen, es sei denn, dass dieses Fragment in den anderen Tonträger in geänderter und beim Hören nicht wiedererkennbarer Form eingefügt wird.

\section{Zur zweiten Frage}

[40] Mit seiner zweiten Frage möchte das vorlegende Gericht wissen, ob Art. 9 Abs. 1 Buchst. b der Richtlinie 2006/115 dahin auszulegen ist, dass es sich bei einem Tonträger, der von einem anderen Tonträger übertragene Musikfragmente enthält, um eine "Kopie" dieses anderen Tonträgers im Sinne dieser Vorschrift handelt. 
[41] Nach Art. 9 Abs. 1 Buchst. b der Richtlinie 2006/115 sehen die Mitgliedstaaten für Tonträgerhersteller ein ausschließliches Recht vor, ihre Tonträger sowie Kopien davon der Öffentlichkeit im Wege der Veräußerung oder auf sonstige Weise zur Verfügung zu stellen.

[42] Weder Art. 9 der Richtlinie 2006/115 noch eine andere Bestimmung dieser Richtlinie definiert den Begriff "Kopie“ im Sinne dieses Artikels.

[43] Der Begriff ist daher unter Berücksichtigung des Regelungszusammenhangs der Bestimmung und des mit der Regelung verfolgten Ziels auszulegen.

[44] Mit dem ausschließlichen Verbreitungsrecht des Tonträgerherstellers aus Art. 9 Abs. 1 Buchst. b der Richtlinie 2006/115 soll diesem durch einen angemessenen Rechtsschutz für seine Rechte des geistigen Eigentums ermöglicht werden, seine Investitionen in die Herstellung von Tonträgern abzusichern, da sich solche Investitionen, wie in den Erwägungsgründen 2 und 5 der Richtlinie 2006/115 ausgeführt, als außerordentlich hoch und risikoreich erweisen können.

[45] Insoweit ergibt sich aus diesem zweiten Erwägungsgrund, dass der dem Tonträgerhersteller durch die Richtlinie gewährte Schutz insbesondere der Bekämpfung der Piraterie dient, d.h., wie der Generalanwalt in Nr. 45 seiner Schlussanträge ausgeführt hat, der Herstellung und öffentlichen Verbreitung nachgeahmter Exemplare von Tonträgern. Die Verbreitung solcher Exemplare stellt nämlich eine besonders schwerwiegende Gefahr für die Interessen des Tonträgerherstellers dar, da sie zu einer erheblichen Verminderung seiner Einkünfte aus der Zurverfügungstellung dieser Tonträger führen kann.

[46] Wie der Generalanwalt in Nr. 46 seiner Schlussanträge festgestellt hat, kann nur ein Gegenstand, der alle oder einen wesentlichen Teil der in einem Tonträger festgelegten Töne übernimmt, aufgrund seiner Merkmale die rechtmäßigen Exemplare des Tonträgers ersetzen und damit eine Kopie dieses Tonträgers im Sinne von Art. 9 Abs. 1 der Richtlinie 2006/115 sein.

[47] Das gilt hingegen nicht für einen Gegenstand, der, ohne alle oder einen wesentlichen Teil der in einem Tonträger festgelegten Töne zu übernehmen, nur Musikfragmente - gegebenenfalls in geänderter Form - aufnimmt, die von diesem Tonträger übertragen werden, um ein neues und davon unabhängiges Werk zu schaffen.

[48] Eine solche Auslegung von Art. 9 Abs. 1 Buchst. b der Richtlinie 2006/115 unter Berücksichtigung seines Ziels wird durch den Zusammenhang bestätigt, in dem die Regelung steht, zu der er gehört.

[49] Wie im siebten Erwägungsgrund der Richtlinie 2006/115 ausgeführt, sollen mit dieser Richtlinie die Rechtsvorschriften der Mitgliedstaaten in der Weise angeglichen werden, dass sie nicht in Widerspruch zu den internationalen Übereinkommen stehen, auf denen das Urheberrecht und die verwandten Schutzrechte in vielen Mitgliedstaaten beruhen.

[50] Zu diesen Übereinkommen gehört das Genfer Übereinkommen, das nach seiner Präambel u.a. zum Ziel hat, auf die weit verbreitete und zunehmende unerlaubte Vervielfältigung von Tonträgern und den Schaden, der dadurch den Interessen der Hersteller von Tonträgern zugefügt wird, zu reagieren.

[51] Das Genfer Übereinkommen enthält in Art. 2 eine Bestimmung, die Art. 9 Abs. 1 Buchst. b der Richtlinie 2006/115 entspricht und speziell vorsieht, dass die Hersteller von Tonträgern gegen die Herstellung von „Vervielfältigungsstücken“ ihrer Tonträger ohne ihre Zustimmung und gegen die Verbreitung dieser Vervielfältigungsstücke an die Öffentlichkeit geschützt werden.

[52] Nach Art. 1 Buchst. c des Genfer Übereinkommens ist ein "Vervielfältigungsstück" ein Gegenstand, der einem Tonträger unmittelbar oder mittelbar entnommene Töne enthält und der „alle oder einen wesentlichen Teil" der in dem Tonträger festgelegten Töne verkörpert.

[53] Die Bestimmungen des Genfer Übereinkommens sind zwar nicht Teil der Unionsrechtsordnung, da die Union zum einen nicht Vertragspartei des Übereinkommens ist und sie zum anderen im Anwendungsbereich des Übereinkommens nicht an die Stelle ihrer Mitgliedstaaten getreten ist - und sei es auch nur, weil nicht alle Mitgliedstaaten Vertragsparteien dieses Übereinkommens sind (vgl. entsprechend Urteil vom 15. März 2012, SCF, C135/10, EU:C:2012:140, Rn. 41). Es ist jedoch eines der in Rn. 49 des vorliegenden Urteils erwähnten internationalen Übereinkommen, so dass die Bestimmungen der Richtlinie 2006/115 so weit wie möglich unter Berücksichtigung dieses Übereinkommens auszulegen sind (vgl. in diesem Sinne Urteile vom 7. Dezember 2006, SGAE, C306/05, EU:C:2006:764, Rn. 35, vom 4. Oktober 2011, Football Association Premier League u.a., C403/08 und C429/08, EU:C:2011:631, Rn. 189, sowie vom 19. Dezember 2018, Syed, C572/17, EU:C:2018:1033, Rn. 20).

[54] Folglich ist in Übereinstimmung mit den Ausführungen des Generalanwalts in den Nrn. 46 und 47 seiner Schlussanträge davon auszugehen, dass der Begriff "Kopie“ iSv Art. 9 Abs. 1 Buchst. b der Richtlinie 2006/115 im Einklang mit dem Begriff „Vervielfältigungsstück“ in Art. 1 Buchst. c und Art. 2 des Genfer Übereinkommens auszulegen ist.

[55] Nach alledem ist auf die zweite Frage zu antworten, dass Art. 9 Abs. 1 Buchst. b der Richtlinie 2006/115 dahin auszulegen ist, dass es sich bei einem Tonträger, der von einem anderen Tonträger übertragene Musikfragmente enthält, nicht um eine 
„Kopie“ dieses anderen Tonträgers im Sinne dieser Vorschrift handelt, da er nicht den gesamten Tonträger oder einen wesentlichen Teil davon übernimmt.

\section{Zur dritten Frage}

[56] Das vorlegende Gericht führt aus, dass nach § 24 Abs. 1 UrhG, der entsprechend für das Recht des Tonträgerherstellers gelte, ein selbständiges Werk, das in freier Benutzung des Werks eines anderen geschaffen worden sei, ohne Zustimmung des Urhebers des benutzten Werks veröffentlicht und verwertet werden dürfe. Dieses „Recht zur freien Benutzung" stelle als solches keine Abweichung vom Urheberrecht dar, sondern bezeichne vielmehr eine dem Urheberrecht immanente Beschränkung seines Schutzbereichs, die auf der Erkenntnis beruhe, dass kulturelles Schaffen nicht ohne ein Aufbauen auf früheren Leistungen anderer Urheber denkbar sei.

[57] Unter diesen Umständen und da sich aus der Antwort auf die zweite Frage ergibt, dass eine Vervielfältigung wie die im Ausgangsverfahren fragliche nicht unter Art. 9 Abs. 1 Buchst. b der Richtlinie 2006/115 fällt, ist davon auszugehen, dass das vorlegende Gericht mit seiner dritten Frage wissen möchte, ob ein Mitgliedstaat in seinem nationalen Recht eine Ausnahme oder Beschränkung in Bezug auf das Recht des Tonträgerherstellers aus Art. 2 Buchst. c der Richtlinie 2001/29 vorsehen darf, die nicht in Art. 5 dieser Richtlinie vorgesehen ist.

[58] Wie sowohl aus der Begründung des Vorschlags für eine Richtlinie des Europäischen Parlaments und des Rates zur Harmonisierung bestimmter Aspekte des Urheberrechts und der verwandten Schutzrechte in der Informationsgesellschaft vom 10. Dezember 1997 (KOM[97] 628 endg.) als auch aus dem 32. Erwägungsgrund der Richtlinie 2001/29 hervorgeht, sind die Ausnahmen und Beschränkungen in Art. 5 dieser Richtlinie erschöpfend aufgeführt, was der Gerichtshof auch mehrfach festgestellt hat (Urteile vom 16. November 2016, Soulier und Doke, C301/15, EU:C:2016:878, Rn. 34, und vom 7. August 2018, Renckhoff, C161/17, EU:C:2018:634, Rn. 16).

[59] Insoweit ist in Rn. 32 des vorliegenden Urteils festgestellt worden, dass die durch die Richtlinie 2001/29 bewirkte Harmonisierung insbesondere vor dem Hintergrund der elektronischen Medien einen angemessenen Ausgleich zwischen den Interessen der Inhaber von Urheber- und verwandten Schutzrechten am Schutz ihres Rechts am geistigen Eigentum auf der einen Seite und dem Schutz der Interessen und Grundrechte der Nutzer von Schutzgegenständen sowie dem Allgemeininteresse auf der anderen Seite sichern soll.

[60] Die Mechanismen, die es ermöglichen, einen angemessenen Ausgleich zwischen diesen verschiedenen Rechten und Interessen zu finden, sind in der Richtlinie 2001/29 selbst verankert, da sie u.a. in den Art. 2 bis 4 die ausschließlichen Rechte der Rechtsinhaber und in Art. 5 die Ausnahmen und Beschränkungen dieser Rechte vorsieht, die von den Mitgliedstaaten umgesetzt werden können oder sogar müssen, wobei diese Mechanismen jedoch durch nationale Maßnahmen zur Umsetzung der Richtlinie und durch deren Anwendung durch die nationalen Behörden zu konkretisieren sind (vgl. in diesem Sinne Urteil vom 29. Januar 2008, Promusicae, C275/06, EU:C:2008:54, Rn. 66 und die dort angeführte Rechtsprechung).

[61] Der Gerichtshof hat wiederholt entschieden, dass die nunmehr in der Charta verankerten Grundrechte, deren Wahrung der Gerichtshof sichert, auf die gemeinsamen Verfassungstraditionen der Mitgliedstaaten sowie auf die Hinweise zurückgehen, die die völkerrechtlichen Verträge über den Schutz der Menschenrechte geben, an deren Abschluss die Mitgliedstaaten beteiligt waren oder denen sie beigetreten sind (vgl. in diesem Sinne Urteil vom 27. Juni 2006, Parlament/Rat, C540/03, EU:C:2006:429, Rn. 35 und die dort angeführte Rechtsprechung).

[62] Zu dem in Rn. 32 des vorliegenden Urteils angesprochenen angemessenen Ausgleich trägt auch Art. 5 Abs. 5 der Richtlinie 2001/29 bei, wonach die in Art. 5 Abs. 1 bis 4 dieser Richtlinie vorgesehenen Ausnahmen und Beschränkungen nur in bestimmten Sonderfällen angewandt werden dürfen, in denen die normale Verwertung des Werks oder eines sonstigen Schutzgegenstands nicht beeinträchtigt wird und die berechtigten Interessen des Rechtsinhabers nicht ungebührlich verletzt werden.

[63] Dabei würde die Wirksamkeit der durch die Richtlinie bewirkten Harmonisierung des Urheberrechts und der verwandten Schutzrechte sowie das mit ihr verfolgte Ziel der Rechtssicherheit gefährdet, wenn jedem Mitgliedstaat ungeachtet des in Rn. 58 des vorliegenden Urteils genannten ausdrücklichen Willens des Unionsgesetzgebers gestattet würde, außerhalb der in Art. 5 der Richtlinie 2001/29 erschöpfend vorgesehenen Ausnahmen und Beschränkungen Abweichungen von den ausschließlichen Rechten des Urhebers aus den Art. 2 bis 4 dieser Richtlinie vorzusehen (Urteil vom 13. Februar 2014, Svensson u. a., C466/12, EU:C:2014:76, Rn. 34 und 35). Aus dem 31. Erwägungsgrund dieser Richtlinie geht nämlich ausdrücklich hervor, dass die Unterschiede, die bei den Ausnahmen und Beschränkungen in Bezug auf bestimmte zustimmungsbedürftige Handlungen bestanden, unmittelbare negative Auswirkungen auf das Funktionieren des Binnenmarkts im Bereich des Urheberrechts und der verwandten Schutzrechte hatten und deshalb die in Art. 5 der Richtlinie 2001/29 aufgeführten Ausnahmen und Beschränkungen die Funktionsfähigkeit des Binnenmarkts sichern sollen.

[64] Außerdem haben die Mitgliedstaaten, wie sich aus dem 32. Erwägungsgrund der Richtlinie ergibt, die Ausnahmen und Beschränkungen in kohärenter Weise anzuwenden. Das Erfordernis der Kohärenz bei der Umsetzung der Ausnahmen und Beschränkungen könnte jedoch nicht gewährleistet werden, wenn es den Mitgliedstaaten freistünde, solche Ausnahmen und Beschränkungen außerhalb der in der Richtlinie 2001/29 ausdrücklich vorgesehenen Ausnahmen anzuordnen (vgl. in 
diesem Sinne Urteil vom 12. November 2015, Hewlett-Packard Belgium, C572/13, EU:C:2015:750, Rn. 38 und 39). Der Gerichtshof hat im Übrigen bereits festgestellt, dass keine Bestimmung der Richtlinie 2001/29 den Mitgliedstaaten die Möglichkeit gibt, die Beschränkungen und Ausnahmen auszuweiten (vgl. in diesem Sinne Urteil vom 10. April 2014, ACl Adam u.a., C435/12, EU:C:2014:254, Rn. 27).

[65] Nach alledem ist auf die dritte Frage zu antworten, dass ein Mitgliedstaat in seinem nationalen Recht keine Ausnahme oder Beschränkung in Bezug auf das Recht des Tonträgerherstellers aus Art. 2 Buchst. c der Richtlinie 2001/29 vorsehen darf, die nicht in Art. 5 dieser Richtlinie vorgesehen ist.

\section{Zur vierten Frage}

[66] Mit seiner vierten Frage möchte das vorlegende Gericht für den Fall, dass ein Eingriff in das ausschließliche Vervielfältigungsrecht des Tonträgerherstellers aus Art. 2 Buchst. c der Richtlinie 2001/29 festgestellt wird, wissen, ob Art. 5 Abs. 3 Buchst. d dieser Richtlinie dahin auszulegen ist, dass der in dieser Bestimmung verwendete Begriff "Zitate" eine Situation erfasst, in der das zitierte Werk nicht zu erkennen ist.

[67] Nach Art. 5 Abs. 3 Buchst. d der Richtlinie 2001/29 können die Mitgliedstaaten für Zitate zu Zwecken wie Kritik oder Rezensionen Ausnahmen und Beschränkungen in Bezug auf die ausschließlichen Rechte zur Vervielfältigung und öffentlichen Wiedergabe aus den Art. 2 und 3 der Richtlinie 2001/29 vorsehen, sofern sie ein Werk oder einen sonstigen Schutzgegenstand betreffen, das bzw. der der Öffentlichkeit bereits rechtmäßig zugänglich gemacht wurde, sofern - außer in Fällen, in denen sich dies als unmöglich erweist - die Quelle, einschließlich des Namens des Urhebers, angegeben wird und sofern die Nutzung den anständigen Gepflogenheiten entspricht und in ihrem Umfang durch den besonderen Zweck gerechtfertigt ist.

[68] Vorab ist in Übereinstimmung mit den Ausführungen des Generalanwalts in den Nrn. 62 und 63 seiner Schlussanträge festzustellen, dass in Anbetracht des Wortlauts von Art. 5 Abs. 3 Buchst. d der Richtlinie 2001/29, der sich auf ein "Werk oder einen sonstigen Schutzgegenstand" bezieht, die in dieser Bestimmung vorgesehene Ausnahme oder Beschränkung auf die Benutzung eines geschützten musikalischen Werks anwendbar ist, sofern die dort vorgesehenen Voraussetzungen erfüllt sind.

[69] Insbesondere verlangt Art. 5 Abs. 3 Buchst. d der Richtlinie 2001/29 für seine Anwendung, wie in Rn. 67 des vorliegenden Urteils ausgeführt, dass die Nutzung „den anständigen Gepflogenheiten entspricht und in ihrem Umfang durch den besonderen Zweck gerechtfertigt ist". Die Nutzung für Zitate darf daher nicht die Grenzen dessen überschreiten, was zur Erreichung des mit dem fraglichen Zitat verfolgten Ziels erforderlich ist.
[70] Da der Begriff des Zitats in der Richtlinie 2001/29 nicht definiert ist, ist seine Bedeutung und Tragweite nach der in Rn. 28 des vorliegenden Urteils angeführten ständigen Rechtsprechung des Gerichtshofs entsprechend seinem Sinn nach dem gewöhnlichen Sprachgebrauch zu bestimmen, wobei zu berücksichtigen ist, in welchem Zusammenhang er verwendet wird und welche Ziele mit der Regelung verfolgt werden, zu der er gehört.

[71] Zum Sinn des Begriffs "Zitat" nach dem gewöhnlichen Sprachgebrauch ist festzustellen, dass die wesentlichen Merkmale eines Zitats darin bestehen, dass ein Werk oder ganz allgemein ein Auszug aus einem Werk von einem Nutzer, der nicht dessen Urheber ist, genutzt wird, um Aussagen zu erläutern, eine Meinung zu verteidigen oder eine geistige Auseinandersetzung zwischen dem Werk und den Aussagen des Nutzers zu ermöglichen, so dass der Nutzer eines geschützten Werks, der sich auf die Ausnahme für Zitate berufen will, das Ziel verfolgen muss, mit diesem Werk zu interagieren, wie der Generalanwalt in Nr. 64 seiner Schlussanträge ausgeführt hat.

[72] Insbesondere kann, wenn der Schöpfer eines neuen musikalischen Werks ein Audiofragment (Sample) nutzt, das einem Tonträger entnommen und beim Hören des neuen Werks wiedererkennbar ist, die Nutzung dieses Audiofragments je nach den Umständen des Einzelfalls ein "Zitat" iSv Art. 5 Abs. 3 Buchst. d der Richtlinie 2001/29 unter Berücksichtigung von Art. 13 der Charta darstellen, sofern die Nutzung zum Ziel hat, mit dem Werk, dem das Audiofragment entnommen wurde, in dem in Rn. 71 des vorliegenden Urteils genannten Sinne zu interagieren, und sofern die Voraussetzungen von Art. 5 Abs. 3 Buchst. d erfüllt sind.

[73] Wie der Generalanwalt in Nr. 65 seiner Schlussanträge hervorgehoben hat, kann es eine solche Interaktion jedoch nicht geben, wenn das zitierte Werk nicht zu erkennen ist.

[74] Nach alledem ist auf die vierte Frage zu antworten, dass Art. 5 Abs. 3 Buchst. d der Richtlinie 2001/29 dahin auszulegen ist, dass der in dieser Bestimmung verwendete Begriff "Zitate" keine Situation erfasst, in der das zitierte Werk nicht zu erkennen ist.

\section{Zur fünften Frage}

[75] Vorab ist darauf hinzuweisen, dass die fünfte Frage, wie sich aus Rn. 24 des vorliegenden Urteils ergibt, insbesondere vor dem Hintergrund gestellt wird, dass das vorlegende Gericht für die Beilegung des Ausgangsrechtsstreits Art. 2 Buchst. c und Art. 5 Abs. 3 Buchst. d der Richtlinie 2001/29 sowie Art. 9 Abs. 1 Buchst. b und Art. 10 Abs. 2 Unterabs. 1 der Richtlinie 2006/115 auszulegen hat.

[76] In diesem Zusammenhang wirft das vorlegende Gericht die Frage auf, ob diese unionsrechtlichen Bestimmungen den Mitgliedstaaten einen Umsetzungsspielraum lassen, da nach 
der Rechtsprechung des Bundesverfassungsgerichts nationale Rechtsvorschriften, die eine Richtlinie der Union umsetzten, grundsätzlich nicht am Maßstab der Grundrechte des Grundgesetzes für die Bundesrepublik Deutschland, sondern allein an den durch das Unionsrecht gewährleisteten Grundrechten zu messen seien, soweit die Richtlinie den Mitgliedstaaten keinen Umsetzungsspielraum überlasse.

[77] In Anbetracht der Antwort auf die zweite und die vierte Frage ist davon auszugehen, dass das vorlegende Gericht mit seiner fünften Frage wissen möchte, ob Art. 2 Buchst. c der Richtlinie 2001/29 dahin auszulegen ist, dass er eine Maßnahme zur vollständigen Harmonisierung darstellt.

[78] Insoweit ist darauf hinzuweisen, dass nach dem Grundsatz des Vorrangs des Unionsrechts, der die Unionsrechtsordnung wesentlich prägt, die Geltung des Unionsrechts in einem Mitgliedstaat nicht dadurch beeinträchtigt werden kann, dass dieser Staat Vorschriften des nationalen Rechts, und haben sie auch Verfassungsrang, geltend macht (Urteil vom 26. Februar 2013, Melloni, C399/11, EU:C:2013:107, Rn. 59).

[79] Da die Umsetzung einer Richtlinie durch die Mitgliedstaaten jedenfalls unter die von Art. 51 der Charta erfasste Situation der Durchführung des Rechts der Union durch die Mitgliedstaaten fällt, muss bei einer solchen Umsetzung das in der Charta vorgesehene grundrechtliche Schutzniveau unabhängig von dem Umsetzungsspielraum der Mitgliedstaaten erreicht werden.

[80] Führt jedoch in einer Situation, in der das Handeln eines Mitgliedstaats nicht vollständig durch das Unionsrecht bestimmt wird, eine nationale Vorschrift oder Maßnahme das Unionsrecht im Sinne von Art. 51 Abs. 1 der Charta durch, steht es den nationalen Behörden und Gerichten weiterhin frei, nationale Schutzstandards für die Grundrechte anzuwenden, sofern durch diese Anwendung weder das Schutzniveau der Charta, wie sie vom Gerichtshof ausgelegt wird, noch der Vorrang, die Einheit und die Wirksamkeit des Unionsrechts beeinträchtigt werden (Urteile vom 26. Februar 2013, Melloni, C399/11, EU:C:2013:107, Rn. 60, und vom 26. Februar 2013, Åkerberg Fransson, C617/10, EU:C:2013:105, Rn. 29).

[81] Daher ist es mit dem Unionsrecht vereinbar, dass die nationalen Gerichte und Behörden die Anwendung dieser nationalen Schutzstandards von dem vom vorlegenden Gericht angeführten Umstand abhängig machen, dass die Bestimmungen einer Richtlinie "Umsetzungsspielräume im nationalen Recht lassen“, sofern sich dies auf den durch die Richtlinienbestimmungen bewirkten Harmonisierungsgrad bezieht, da die Anwendung nationaler Schutzstandards nur in Betracht kommt, soweit die Bestimmungen keine vollständige Harmonisierung bewirken.

[82] Vorliegend ist festzustellen, dass die Richtlinie 2001/29 die Harmonisierung nur bestimmter Aspekte des Urheberrechts und der verwandten Schutzrechte zum Ziel hat und außerdem einige ihrer Bestimmungen die Absicht des Unionsgesetzge- bers erkennen lassen, den Mitgliedstaaten einen Umsetzungsspielraum einzuräumen (vgl. in diesem Sinne Urteil vom 5. März 2015, Copydan Båndkopi, C463/12, EU:C:2015:144, Rn. 57).

[83] Zum ausschließlichen Recht der Urheber aus Art. 2 Buchst. c der Richtlinie 2001/29 ist in Rn. 27 des vorliegenden Urteils darauf hingewiesen worden, dass die Mitgliedstaaten nach dieser Bestimmung für die Tonträgerhersteller in Bezug auf ihre Tonträger das ausschließliche Recht vorsehen, die „unmittelbare oder mittelbare, vorübergehende oder dauerhafte Vervielfältigung auf jede Art und Weise und in jeder Form ganz oder teilweise zu erlauben oder zu verbieten".

[84] In dieser Bestimmung wird somit das ausschließliche Vervielfältigungsrecht der Hersteller von Tonträgern in der Union eindeutig festgelegt. Die Bestimmung ist zudem an keine Bedingung geknüpft, und es bedarf zu ihrer Durchführung oder Wirksamkeit auch keiner weiteren Maßnahme.

[85] Art. 2 Buchst. c der Richtlinie 2001/29 stellt demnach eine Maßnahme zur vollständigen Harmonisierung des materiellen Gehalts des in ihm geregelten Rechts dar (vgl. entsprechend zum ausschließlichen Recht des Inhabers einer Unionsmarke Urteile vom 20. November 2001, Zino Davidoff und Levi Strauss, C414/99 bis C416/99, EU:C:2001:617, Rn. 39, sowie vom 12. November 2002, Arsenal Football Club, C206/01, EU:C:2002:651, Rn. 43).

[86] Nach alledem ist auf die fünfte Frage zu antworten, dass Art. 2 Buchst. c der Richtlinie 2001/29 dahin auszulegen ist, dass er eine Maßnahme zur vollständigen Harmonisierung des materiellen Gehalts des darin geregelten Rechts darstellt.

\section{Kosten}

[87] Für die Parteien des Ausgangsverfahrens ist das Verfahren ein Zwischenstreit in dem bei dem vorlegenden Gericht anhängigen Rechtsstreit; die Kostenentscheidung ist daher Sache dieses Gerichts. Die Auslagen anderer Beteiligter für die Abgabe von Erklärungen vor dem Gerichtshof sind nicht erstattungsfähig.

Aus diesen Gründen hat der Gerichtshof (Große Kammer) für Recht erkannt:

1. Art. 2 Buchst. c der Richtlinie 2001/29/EG des Europäischen Parlaments und des Rates vom 22. Mai 2001 zur Harmonisierung bestimmter Aspekte des Urheberrechts und der verwandten Schutzrechte in der Informationsgesellschaft ist unter Berücksichtigung der Charta der Grundrechte der Europäischen Union dahin auszulegen, dass das ausschließliche Recht des Tonträgerherstellers aus dieser Bestimmung, die Vervielfältigung seines Tonträgers zu erlauben oder zu verbieten, ihm gestattet, sich dagegen zu wehren, dass ein Dritter ein - auch nur sehr kurzes - Audiofragment seines Tonträgers nutzt, um es in einen anderen Tonträger 
einzufügen, es sei denn, dass dieses Fragment in den anderen Tonträger in geänderter und beim Hören nicht wiedererkennbarer Form eingefügt wird.

2. Art. 9 Abs. 1 Buchst. b der Richtlinie 2006/115/EG des Europäischen Parlaments und des Rates vom 12. Dezember 2006 zum Vermietrecht und Verleihrecht sowie zu bestimmten dem Urheberrecht verwandten Schutzrechten im Bereich des geistigen Eigentums ist dahin auszulegen, dass es sich bei einem Tonträger, der von einem anderen Tonträger übertragene Musikfragmente enthält, nicht um eine „Kopie" dieses anderen Tonträgers im Sinne dieser Vorschrift handelt, da er nicht den gesamten Tonträger oder einen wesentlichen Teil davon übernimmt.

3. Ein Mitgliedstaat darf in seinem nationalen Recht keine Ausnahme oder Beschränkung in Bezug auf das Recht des Tonträgerherstellers aus Art. 2 Buchst. c der Richtlinie 2001/29 vorsehen, die nicht in Art. 5 dieser Richtlinie vorgesehen ist.

4. Art. 5 Abs. 3 Buchst. d der Richtlinie 2001/29 ist dahin auszulegen, dass der Begriff "Zitate“ in dieser Bestimmung keine Situation erfasst, in der das zitierte Werk nicht zu erkennen ist.

5. Art. 2 Buchst. c der Richtlinie $2001 / 29$ ist dahin auszulegen, dass er eine Maßnahme zur vollständigen Harmonisierung des materiellen Gehalts des in ihm geregelten Rechts darstellt.

Lenaerts, Arabadjiev, Vilaras, von, Danwitz, Toader, Biltgen, Lycourgos, Juhász, Ilešič, Bay, Larsen, Rodin

Verkündet in öffentlicher Sitzung in Luxemburg am 29. Juli 2019.

Der Kanzler: A. Calot Escobar

Der Präsident: $K$. Lenaerts

Verfahrenssprache: Deutsch. 